See discussions, stats, and author profiles for this publication at: https://www.researchgate.net/publication/221757737

\title{
First and second order statistical texture features in carotid plaque image analysis: Preliminary results from ongoing research
}

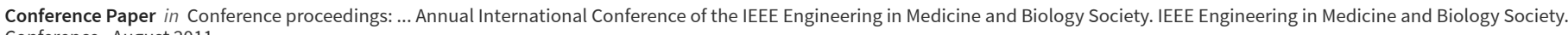
Conference · August 2011

DOI: 10.1109/IEMBS.2011.6091641 · Source: PubMed

CITATIONS

2

8 authors, including:

Efthyvoulos C Kyriacou

Frederick University

174 PUBLICATIONS 2,434 CITATIONS

SEE PROFILE

C. S. Pattichis

University of Cyprus

431 PUBLICATIONS 5,663 CITATIONS

SEE PROFILE
READS

69

Ardrew Nicolaides

St George's, University of London/University of Nicosia Medical Schoool, Nicosia, Cy...

875 PUBLICATIONS 27,186 CITATIONS

SEE PROFILE

7. Styliani Petroudi

University of Cyprus

55 PUBLICATIONS 448 CITATIONS

SEE PROFILE

Some of the authors of this publication are also working on these related projects:

Dynamically Reconfigurable Architecture Systems for Time-Varying Image Constraints (DRASTIC) View project

eENERCA - European Network for Rare and Congenital Anaemias View project 


\title{
First and Second Order Statistical Texture Features in Carotid Plaque Image Analysis: Preliminary Results From Ongoing Research
}

\author{
Efthyvoulos Kyriacou, Senior Member, IEEE, Andrew Nicolaides, \\ Constantinos S. Pattichis, Senior Member, IEEE, Styliani Petroudi, Member IEEE \\ Marios Pattichis, Senior Member, IEEE, Maura Griffin, Stavros Kakkos, Grigoris Makris
}

\begin{abstract}
Carotid plaques have been associated with ipsilateral neurological symptoms. High-resolution ultrasound can provide information not only on the degree of carotid artery stenosis but also on the characteristics of the arterial wall including the size and consistency of atherosclerotic plaques. The aim of this study was to determine cerebrovascular risk stratification based on ultrasonic plaque texture features and clinical features in patients with asymptomatic internal carotid artery (ICA) stenosis. It is shown that cerebrovascular risk stratification is possible using a combination of clinical and ultrasonic plaque features with very satisfactory results. However, these findings need to be validated in additional prospective studies in patients having current medical intervention.
\end{abstract}

\section{INTRODUCTION}

$\mathrm{S}$ tudies performed in the 1980s and 1990s [1] have indicated that the risk of stroke in asymptomatic patients is $0.1-1.6 \%$ per year for internal carotid artery (ICA) stenosis $<75-80 \%$ (NASCET method i.e. in relation to the diameter of the normal distal internal carotid artery) and $2.0-3.3 \%$ per year with greater degrees of stenosis. Two randomized controlled trials, the ACAS in 1995 [2] and the ACST in 2004 [3] reported that in patients with asymptomatic ICA stenosis $>60-70 \%$ (NASCET) carotid endarterectomy reduced the risk of stroke from $2 \%$ to $1 \%$ per year [3], [4]. In these trials carotid endarterectomy was associated with a $2-3 \%$ perioperative rate of stroke or death.

E.C.Kyriacou is with the Frederick University, Limassol, Cyprus. (email: e.kyriacou@frederick.ac.cy). Prof. Kyriacou is the corresponding author.

A. Nicolaides is Prof. Emeritus at Imperial College London, UK while currently is with the Vascular screening and Diagnostic Centre, London, UK, and with the Cyprus Cardiovascular Disease Educational research Trust, Nicosia, Cyprus. (email: andisnicolai@gmail.com).

C. Pattichis and S. Petroudi are with the Department of Computer Science, University of Cyprus, Nicosia, Cyprus. (email: \{pattichi,styliani\}@ucy.ac.cy).

M. Pattichis is with the Department of electrical and Computer engineering, University of New Mexico, New Mexico, USA. (email: pattichis@eece.unm.edu)

S. Kakkos is with the Department of Vascular Surgery, University of Patras Medical School, Patras, Greece. (email: kakkosstavros@gmail.com)

M. Griffin is with the Vascular Noninvasive Screening and Diagnostic Centre, London, UK. (email: maurabgriffin@googlemail.com)

G. Makris is with Imperial College, London UK. (email: g.makris09@imperial.ac.uk)
However, medical intervention which was left to the discretion of the local teams was suboptimal in relation to current practice.

Currently, vascular disease medical intervention includes ongoing risk factor diagnosis, patient education, support of healthy life style practices and medications. Best evidence indicates that the average annual risk of ipsilateral cerebral and any territory stroke among patients with asymptomatic severe ICA stenosis receiving medical intervention alone has fallen to approximately $1 \%$ [5], [6] making routine carotid endarterectomy unjustified. However, if patient subgroups with sufficiently higher than average risk, despite current optimal medical intervention, could be reliably identified, then carotid surgery may still be justified.

In addition it has been proved that high-resolution ultrasound provides information not only on the degree of carotid artery stenosis but also on the characteristics of the arterial wall including the size and consistency of atherosclerotic plaques. Several studies have indicated that "complicated" carotid plaques are often associated with ipsilateral neurological symptoms and share common ultrasonic characteristics, being more echolucent (weak reflection of ultrasound and therefore containing echo-poor structures) and heterogeneous (having both echolucent and echogenic areas). In contrast, "uncomplicated" plaques which are often asymptomatic tend to be of uniform consistency (uniformly hypoechoic or uniformly hyperechoic) without evidence of ulceration ([1], [8], [9], [10], [14]). Different classifications of plaque ultrasonic appearance have been proposed in the literature. Reilly classified [1] carotid plaques as homogenous and heterogeneous, defining as homogeneous plaques those with "uniformly bright echoes" that are now known as uniformly hyperechoic (type 4).Johnson (1985) [12] classified plaques as dense and soft. Widder (1990) [13] classified them as echolucent and echogenic based on the their overall level of echo patterns. Gray-Weale (1988) [11] suggested 4 types: (i) type 1: predominantly echolucent lesions, (ii) type 2: echogenic lesions with substantial (>75\%) components of echolucency, (iii) type 3: predominately echogenic with small area(s) of echolucency occupying less than a quarter of the plaque, and (iv) type 4: uniformly dense echogenic lesions. Geroulakos (1993) [14] subsequently modified the Gray-Weale classification by using a $50 \%$ area cut off point 
instead of $75 \%$ and by adding a fifth type, which as a result of heavy calcification on its surface cannot be correctly classified.

Having in mind all the above, this paper presents the steps for creating a method for cerebrovascular risk stratification based on clinical factors and image analysis. The data were collected through a multicentre cohort study of patients with asymptomatic ICA stenosis called the Asymptomatic Carotid Stenosis and Risk of Stroke Study (ACSRS) [1].

\section{METHODS}

\section{A. Patient Recruitment}

Newly referred ( $<3$ months) patients with 50-99\% ICA stenosis in relation to the carotid bulb diameter (ECST method) without previous ipsilateral cerebral or retinal ischemic (CORI) symptoms and without neurological abnormality were recruited to the study after written informed consent. Patients who had had contralateral cerebral hemispheric/retinal or vertebrobasilar symptoms or signs of stroke/TIA were included if asymptomatic for at least 6 months prior to recruitment. The ratio of recruited patients with stenosis $50-69 \%$ and $70-79 \%$ (ECST) from each centre was 1:2. For patients with bilateral carotid atherosclerosis the side with the more severe stenosis was considered ipsilateral (the study artery).

\section{B. Clinical and Biochemical Characteristics}

At baseline all patients had a history taken and a physical examination by the local neurologist, electrocardiographic (ECG) examination and collection of fasting blood for determination of the following:

-Age, gender, body mass index (BMI), systolic and diastolic blood pressure, smoking history and accrued packyears.

-Medication usage including antiplatelet, anti-hypertensive and lipid lowering agents.

-Presence of hypertension (antihypertensive medication or $\mathrm{BP} \geq 140 \mathrm{mmHg}$ systolic or $\geq 90 \mathrm{mmHg}$ diastolic); coronary artery disease (documented myocardial infarction/angina, coronary artery bypass or stenting); diabetes (antihyperglycemic therapy or fasting blood glucose $>120$ $\mathrm{mg} / \mathrm{dL}$ ) and previous contralateral stroke/TIA or vertebrobasilar symptoms.

-ECG evidence of atrial fibrillation, previous myocardial infarction (MI), myocardial ischemia and left ventricular hypertrophy (LVH) on baseline ECG according to previously published criteria. ECGs were reported at the coordinating centre by two cardiologists.

-Fibrinogen, fasting lipids (total cholesterol, HDLcholesterol, LDL-cholesterol, triglycerides), serum creatinine and hematocrit.

\section{Duplex Scanning}

Bilateral carotid duplex scanning was performed on admission to the study. Ultrasonographers from all centers were trained at the coordinating centre in grading internal carotid stenosis and plaque image capture. The entire duplex examination, recorded on S-VHS videotape, was sent to the coordinating centre for quality control.

Images from video recordings were digitized off-line on a PC using a video grabber card. Image normalization for gray scale using linear scaling with "blood" (gray scale $=0$ ) and adventitia (gray scale $=190$ ), and pixel density standardization to 20 pixels per $\mathrm{mm}$ were performed followed by image analysis. The "Plaque Texture Analysis software" version 3.2 (Iconsoft International Ltd, PO Box 172, Greenford, London UB6 9ZN, UK) [15] was used. Plaques were segmented by expert physicians using the same software [1], [16].

\section{Image Analysis}

Several image texture analysis features were extracted and used in the statistical analysis [16], [17]. These features have been presented in previous studies. The algorithms used namely are: 1) Statistical Features (SF), 2) Spatial Gray Level Dependence Matrices (SGLDM) algorithm, 3) Gray Level Difference Statistics (GLDS) algorithm, 4) Neighbourhood Gray Tone Difference Matrix (NGTDM), 5)Statistical Feature Matrix (SFM) method, 6) Laws Texture Energy Measures (TEM), 7) Fractal Dimension Texture Analysis (FDTA), 8) Fourier Power Spectrum (FPS), 9) Run Length Statistics (RUNL), and10)Plaque Shape Features (SHAPE).

\section{E. Patient Data}

A total of 1121 patients between 39 and 89 years (mean age $70.0 \pm$ SD 7.7, 61\% male) were recruited during 19982002 with a follow-up of 6-96 months (mean 48 months) in the context of the ACSRS study. A total of 130 first ipsilateral CORI events occurred (59 strokes of which 12 were fatal, 49 TIAs and 22 amaurosis fugax). There were 49 first contralateral CORI events (18 ischemic strokes of which 7 were fatal, 22 TIAs and 9 amaurosis fugax). There were 2 vertebrobasilar strokes.

There have been a total of 214 deaths (195 non-stroke deaths) of which $157(73 \%)$ were due to cardiovascular causes: myocardial infarction 110, fatal stroke 19 (12 ipsilateral and 7 contralateral already mentioned above), heart failure 17, pulmonary embolism 3, lower limb ischemia/gangrene 3, ruptured abdominal aortic aneurysm 3, renal failure 1 and mesenteric artery thrombosis 1 . There were 56 non-vascular deaths; malignancy 37 , pneumonia/respiratory failure 12, gastrointestinal hemorrhage 2, dementia 2, road traffic accident 2 and general surgical procedure 1. Cause of death was unknown in one patient.

\section{RESULTS}

Analysis was done using the Cox Proportional Hazard Model [18] based on the following steps:

Step1: For each set of texture features, ROC curves for events were constructed and texture features with ROC Area Under the Curve (AUC) in the range of 0.65-0.35 were 


\begin{tabular}{lrrrrr}
\multicolumn{5}{c}{ TABLE I Variables used in the equation of the COX model (see text in III. Results for variables explanation) } \\
\hline & \multicolumn{1}{c}{} & \multicolumn{1}{c}{ SE } & Wald & df & \multicolumn{1}{c}{ Sig. } \\
\hline SGLDM_SVAR_mean & -.011 & .008 & 2.024 & 1 & .155 \\
SGLDM_diff_var_range & 4.002 & 2.472 & 2.622 & .989 \\
Ln(SGLDM_con_mean) & .600 & .137 & 19.054 & 1 & .105 \\
Ln(SGLD_ent_mean) & 2.516 & 1.166 & 4.658 & 1 & .000 \\
SGLDM_SAV_range & -17.443 & 8.160 & 4.570 & 1 & .031 \\
CUBRAR & .574 & .202 & 8.081 & 1.821 \\
CTIASTR1 & .702 & .285 & 6.049 & 1 & .033 \\
\hline
\end{tabular}

TABLE II 5 year stroke rate prediction groups (into 6 classes (a.), and 3 classes (b.)).

\begin{tabular}{|c|c|c|c|}
\hline $\begin{array}{l}\text { Group } \\
\text { (Predicted risk) }\end{array}$ & $\begin{array}{l}\text { Number } \\
\text { of } \\
\text { patients }\end{array}$ & $\begin{array}{l}\text { Number } \\
\text { of } \\
\text { Strokes }\end{array}$ & $\begin{array}{l}\text { 5-YR } \\
\text { Stroke rate } \\
\text { (Observed) }\end{array}$ \\
\hline $0(0-5 \%)$ & 682 & 7 & $2 \%$ \\
\hline $1(5-10 \%)$ & 172 & 6 & $5 \%$ \\
\hline $2(10-20 \%)$ & 148 & 20 & $17 \%$ \\
\hline $3(20-30 \%)$ & 49 & 12 & $32 \%$ \\
\hline $4(30-40 \%)$ & 28 & 6 & $27 \%$ \\
\hline $5(>40 \%)$ & 13 & 6 & $45 \%$ \\
\hline
\end{tabular}

b. \begin{tabular}{lllr}
\hline $\begin{array}{l}\text { Group } \\
\text { (Predicted risk) }\end{array}$ & $\begin{array}{l}\text { Number } \\
\text { of } \\
\text { patients }\end{array}$ & $\begin{array}{l}\text { Number } \\
\text { of } \\
\text { Strokes }\end{array}$ & $\begin{array}{l}\text { 5-YR } \\
\text { Stroke rate } \\
\text { (Observed) }\end{array}$ \\
\hline $0(0-10 \%)$ & 854 & 13 & $3 \%$ \\
$1(10-20 \%)$ & 148 & 20 & $17 \%$ \\
$2(>20 \%)$ & 90 & 24 & $34 \%$ \\
\hline
\end{tabular}

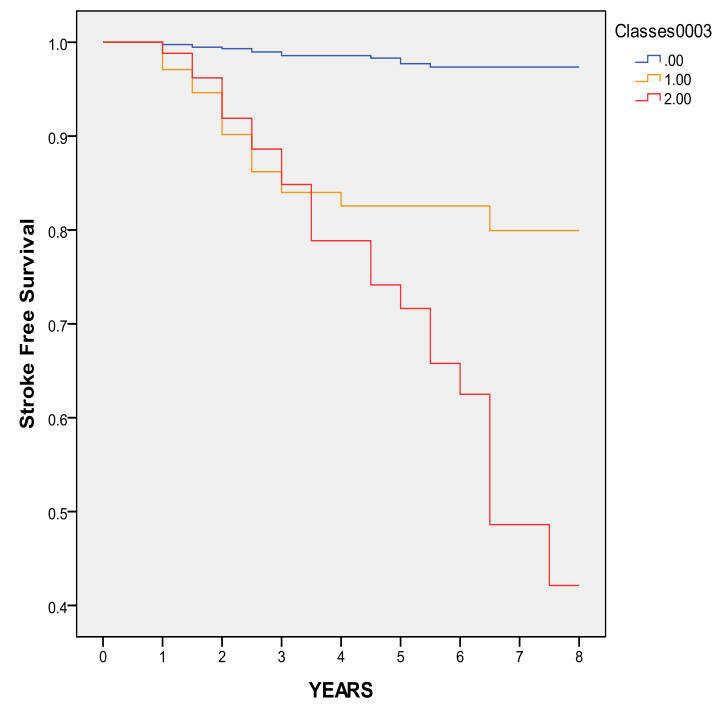

Fig.1 Stroke free survival for three classes using the COX model tabulated in Table I with stroke as dependent variable. The three classes are given in Table IIb, where 0,1 , and 2 correspond to $(0-10 \%)$, $(10-20 \%)$, and $(>20 \%)$ respectively.

discarded. Only features with AUC $>0.65$ or $<0.35$ were used in subsequent analysis. Those not normally distributed were normalised.

Step 2: A linear logistic analysis was performed with stroke as the dependent variable in order to identify and include the significant variables in the model (see Table I).

The best variables selected and used in the model as tabulated in Table I are: SGLDM_SVAR_mean; which is the mean value of the feature Sum Variance from the SGLDM algorithm. SGLDM_diff_var_range; which is the range of the feature difference variance from the SGLDM algorithm. In(SGLDM_con_mean); which is the natural logarithm of the mean value of the feature Contrast from the SGLDM Algorithm. $\ln$ (SGLDM_ent_mean); which is the natural logarithm of the mean value of the feature Entropy Mean from the SGLDM Algorithm. SGLDM_SAV_range; which is the range of the feature Sum Average from the SGLDM algorithm. CUBRAR which is the cube root of the total plaque area. CTIASTR1 is the history of TIAs and strokes. The Area Under the Curve ROC curves was 0.834 (0.775 to 0.893).

For stroke as the dependent variable the explanatory variables are significant with a $94.7 \%$ correct classification.

Using the above variables a Cox model was run for stroke as the dependent variable. In order to calculate the predicted five year stroke risk for each patient we are following the procedure as described by Nicolaides et al. [1]:

Step 1: Subtract mean covariates from baseline covariates for each individual to find difference $\mathrm{x}$.

Step 2: Multiply $\mathrm{x}$ with $\beta$ to derive $\beta \mathrm{x}$.

Step3: For every patient calculate SUM of the $\beta \mathrm{xs}$ i.e. $\Sigma \beta \mathrm{x}$.

Step4: For every patient predicted 5 year survival probability $=100 * 0.963^{\exp (\Sigma \beta \mathrm{x})}$

Step5: Calculate 5 year predicted stroke rate and average annual stroke rate.

Step6: Group patients into classes of predicted stroke rate and compare with observed event rate using life-table analysis of these groups.

Images were divided into six different groups as displayed in Table IIa. In order to improve the percentage we regrouped to a smaller set of three different groups as newgroup $0=$ initialgroups $(0+1)$, newgroup $1=$ initialgroup(2), newgroup $2=$ initialgroups $(3+4+5)$ (see Table IIb and Fig. 1).

\section{CONCLUDING REMARKS}

The ACSRS is the largest prospective study of patients with asymptomatic carotid artery stenosis undergoing 
medical intervention alone. The preliminary results demonstrate that a number of baseline clinical characteristics and ultrasonic plaque features are independent predictors of subsequent ipsilateral CORI events. Certain findings cannot be explained. For example the discrepancy between predicted risk and observed risk in group 4 in table II, Different Cox models are being explored

This study is unique not only because of the relatively large number of patients studied but also because, in contrast to previous studies that had concentrated on one feature only, it shows how plaque characteristics can add significantly to the improvement of risk stratification. It also provides a method that allows estimation of risk for any patient.

Ultrasonic imaging is to a certain extent operator dependent. This can be overcome by training ultrasonographers in equipment presets and image capture; also, by performing image normalization with computerized analysis. The importance of training vascular ultrasonographers in equipment settings and plaque imaging for optimal results cannot be overemphasized.

A limitation of the ACSRS study was that the medical management of patients was according to what was considered best medical therapy at each centre at the time. At each centre the clinician in charge was free to change therapy according to changing indications. At the beginning of the study only $84 \%$ of patients were on antiplatelet therapy and only $25 \%$ on lipid lowering therapy. Towards the end of the study these percentages were $95 \%$ and $85 \%$ respectively. However, the intensity of the treatment varied and unlike current guidelines very few patients were treated to target cholesterol level. In addition, this "freedom" in management resulted in $129(11.5 \%)$ patients having a carotid endarterectomy in the absence of symptoms soon after the results of the ACST were published. Despite this, follow-up to a CORI event, death or to the end of the study was achieved in $87 \%$ of patients.

The clinical implication of the ACSRS study is that clinical and ultrasonic plaque features can be used to stratify risk and may lead to refinement of the indications for carotid endarterectomy. The availability of user friendly software for image analysis and automatic calculation of risk can make the method part of routine practice in the vascular laboratory.

Validation of predicted risk in this study was limited since this was done internally, that is for the same group of patients on whom the score was developed. The findings need to be validated in additional prospective observational studies using current medical intervention or in the medical arm of randomized controlled trials comparing carotid endarterectomy plus medical intervention against medical intervention alone.

\section{REFERENCES}

[1] A. Nicolaides, S. Kakkos, E. Kyriacou, M. Griffin et al. "Asymptomatic Internal Carotid Artery Stenosis and Cerebrovascular Risk Stratification," Journal of Vascular Surgery, vol. 52, No.4 pp. 1486-1496, Dec. 2010.
[2] Executive Committee for the Asymptomatic Carotid Atherosclerosis Study, "Endarterectomy for asymptomatic carotid artery stenosis," J. Am Med Assoc, vol.273, pp.1421-8, 1995.

[3] MRC Asymptomatic Carotid Surgery Trial (ACST) Collaborative Group, "Prevention of disabling and fatal strokes by successful carotid endarterectomy in patients without recent neurological symptoms: randomized controlled trial," Lancet, vol. 363, pp.1491502, 2004.

[4] Executive Committee for the Asymptomatic Carotid Atherosclerosis Study, "Endarterectomy for asymptomatic carotid artery stenosis," J. Am Med Assoc, vol.273, pp.1421-8, 1995.

[5] A.R. Naylor, P.A. Gaines, P.M. Rothwell, "Who benefits most from intervention in asymptomatic carotid stenosis: patients or professionals?," Eur J Vasc Endovasc Surg, vol.37, pp. 625-32, 2009.

[6] A.L. Abbott, "Medical (non-surgical) intervention alone is now best for prevention of stroke associated with asymptomatic severe carotid stenosis: results of a systematic review and analysis," Stroke, vol.40, pp.573-583, 2009.

[7] L.M. Reilly, R.J. Lusby, L. Hughes, L., et al., "Carotid plaque histology using real-time ultrasonography. Clinical and therapeutic implications," American Journal of Surgerym, vol.146, pp.188-93, 1983.

[8] T.F. O'Donnell Jr., L. Erdoes, W.C. Mackey et al. , "Correlation of Bmode ultrasound imaging and arteriography with pathologic findings at carotid endarterectomy," Archives of Surgery, vol.120, pp.443-9,1985.

[9] A.L. Leahy, P.T. McCollum, T.M. Feeley et al., "Duplex ultrasonography and selection of patients for carotid endarterectomy: Plaque morphology or luminal narrowing?," Journal of Vascular Surgery, vol.8, pp.558-62, 1998.

[10] M. Langsfeld, A.C. Gray-Weale, R.J. Lusby, "The role of plaque morphology and diameter reduction in the development of new symptoms in asymptomatic carotid arteries," Journal of Vascular Surgery, vol.9, pp.548-57, 1989.

[11] A.C. Gray-Weale, J.C. Graham, J.R. Burnett et al., "Carotid artery atheroma: comparison of preoperative B-mode ultrasound appearance with carotid endarterectomy specimen pathology," Journal of Cardiovascular Surgery, vol.29,pp. 676-81, 1988.

[12] J.M. Johnson, M.M. Kennelly, D. Decesare, et al., "Natural history of asymptomatic carotid plaque," Archives of Surgery, vol.120, pp.10102,1985 .

[13] B. Widder, K. Paulat, J. Hachspacher, et al., "Morphological characterization of carotid artery stenoses by ultrasound duplex scanning," Ultrasound in Medicine and Biology, vol.16, pp.349-54, 1990.

[14] G. Geroulakos, G. Ramaswami, A. Nicolaides, et al., "Characterisation of symptomatic and asymptomatic carotid plaques using highresolution real-time ultrasonography," British Journal of Surgery, vol.80, pp.1274-7, 1993.

[15] M. Griffin, A.Nicolaides, E.Kyriacou, "Normalisation of ultrasonic images of atherosclerotic plaques and reproducibility of grey scale median using dedicated software," Int Angiol, vol.26, pp.372-7, 2007.

[16] S.K. Kakkos, J.M. Stevens, A.N. Nicolaides, E. Kyriacou, C.S.Pattichis, G. Geroulakos, D. Thomas, "Texture Analysis of Ultrasonic Images of Symptomatic Carotid Plaques Can Identify Those Plaques Associated with Ipsilateral Embolic Brain Infarction," European Journal of Vascular and Endovascular Surgery, vol.33, no4, pp.422-429, 2007.

[17] C.I. Christodoulou, C.S. Pattichis, M. Pantziaris, A. Nicolaides, "Texture Based Classification of Atherosclerotic Carotid Plaques," IEEE Transactions on Medical Imaging, vol.22, no7, pp.902912,2003

[18] D. R. Cox and D. Oakes , "Analysis of survival data," Chapman \& Hall, 1984. 